\title{
Vertical Displacement due to Ocean Tidal Loading Around Taiwan Based on GPS Observations
}

\author{
Ta-Kang Yeh $^{1, *}$, Cheinway Hwang ${ }^{2}$, Jiu-Fu Huang ${ }^{2}$, Benjamin Fong Chao ${ }^{3}$, and Ming-Han Chang ${ }^{4}$ \\ ${ }^{I}$ Department of Real Estate and Built Environment, National Taipei University, Taipei, Taiwan, ROC \\ ${ }^{2}$ Department of Civil Engineering, National Chiao Tung University, Hsinchu, Taiwan, ROC \\ ${ }^{3}$ Institute of Earth Sciences, Academia Sinica, Taipei, Taiwan, ROC \\ ${ }^{4}$ Institute of Geomatics and Disaster Prevention Technology, Ching Yun University, Jhongli, Taiwan, ROC
}

Received 28 October 2010, accepted 27 January 2011

\begin{abstract}
Ocean tidal loading (OTL) is an important factor in GPS positioning, especially along the vertical direction. OTL influences the precision of GPS positioning and produces height variations of up to $12 \mathrm{~cm}$. In this study, daily GPS data obtained from 27 GPS tracking stations around Taiwan were collected, and four OTL models were applied to relative static GPS positions derived from these data. The GPS data were obtained from 8 - 14 August 2006 (summer) and 1 - 30 January 2007 (winter). The software Bernese 5.0 was utilized for data processing.

During the summer, the average GPS error along the vertical axis was $2.72 \mathrm{~cm}$. After correcting the measured heights with the Schwiderski, NAO.99b, CSR4.0 and TPXO.6.2 models, the average errors became 2.30, 2.30, 2.30 and $2.31 \mathrm{~cm}$, respectively. In winter, the average GPS error in the vertical direction was $1.32 \mathrm{~cm}$. After correcting the heights using the above models, the average errors became $0.86,0.85,0.89$ and $0.84 \mathrm{~cm}$, respectively. The precision of the vertical direction was improved by approximately $15-36 \%$ on average.

In eastern Taiwan, all GPS stations in the vertical direction have amplitudes smaller than $1 \mathrm{~cm}$, because the ocean is more than $2000 \mathrm{~m}$ deep and with less shallow and narrow bights and estuaries. On the other hand, the amplitudes of GPS stations in northwestern Taiwan are larger than $1 \mathrm{~cm}$ because the ocean is only approximately $200 \mathrm{~m}$ deep. The amplitudes in the vertical direction of GPS stations are approximately $1.2-2.7 \mathrm{~cm}$ on the offshore islands of Taiwan, larger than average in Taiwan. In the future, local corrections from an OTL model are necessary for high accurate GPS positioning.
\end{abstract}

Key words: GPS, Ocean tidal loading, Vertical displacement, Taiwan

Citation: Yeh, T. K., C. Hwang, J. F. Huang, B. F. Chao, and M. H. Chang, 2011: Vertical displacement due to ocean tidal loading around Taiwan based on GPS observations. Terr. Atmos. Ocean. Sci., 22, 373-382, doi: 10.3319/TAO.2011.01.27.01(T)

\section{INTRODUCTION}

The Global Positioning System (GPS) is extensively used in diverse applications such as surveying, cadastral surveying, fault movement detection and geodynamics because of its efficiency, flexibility and high accuracy. However, GPS often incurs systematic errors because the surrounding environment can adversely impact carrier phases. The factors that contribute to reduced GPS accuracy include tropospheric delay, ionospheric delay, antenna phase center variations, GPS satellite and receiver clock errors, cycle slips and multi-path effects (Xu 2007).

\footnotetext{
* Corresponding author

E-mail:bigsteel@cyu.edu.tw
}

Although capable of resolving these systematic errors, conventional correction methods fail to address GPS positioning inaccuracy caused by ocean tidal loading (OTL). Ocean tides refer to the rise and fall of ocean waves according to certain cycles caused by gravitational forces from celestial bodies. Tides rise and fall twice daily, and the duration of each cycle depends on the place of observation. Tidal phenomena are dominated by the gravitational attraction of the Moon and to a lesser extent the Sun. Ocean tidal loading has received extensive attention in the literature. According to Baker et al. (1995), the vertical amplitude of OTL in Newlyn, UK, can be as high as $12 \mathrm{~cm}$ and this value denotes the vertical deformation of the surface of the Earth. 
Dragert et al. (2000) indicated that in Holberg, along the Canadian seashore, the amplitude of OTL can reach $8 \mathrm{~cm}$. Jentzsch et al. (2000) found the strong tidal anomalies due to ocean tidal loading on Greenland. The loading computations confirmed the observations, but it turned out that with global models only about $50 \%$ of the observed effect can be explained. This means that at these GPS stations a vertical deformation of up to $\pm 3.5 \mathrm{~cm}$ is not corrected applying the global models. Penna and Baker (2002) investigated OTL along the northwestern coast of Australia, where variations in the vertical coordinate can be as high as $10 \mathrm{~cm}$ and have an oscillation period of approximately 6 hours, likely resulting from semi-diurnal tides. Additionally, Khan and Scherneck (2003) analyzed approximately $500 \mathrm{~km}$ of the Alaskan coast of the United States and verified an OTL model with GPS and gravity data. From a global perspective, Zahran et al. (2005) indicated that the displacement of inland stations due to OTL has a baseline variation of $2 \mathrm{~cm}$ and can be as high as $2 \mathrm{~cm}$, demonstrating that although OTL effects are strongest near the coast, they can extend far inland (Yeh et al. 2008).

Moreover, Bos and Scherneck (2007) designed a webpage that allows users to easily generate OTL predictions from several high-quality ocean tide models at no charge. The Physical Oceanography Distributed Active Archive Center (PODAAC), under the leadership of NASA's Jet Propulsion Laboratory, also provides a related online service (PODAAC 2007). Addressing the increasing theoretical attention paid to oceanic and earth tides, a technological report published by the International Earth Rotation Service (IERS) includes a chapter on tidal corrections for high-precision positioning (van Dam et al. 2003). Therefore, surveying in Taiwan should account for the effects of OTL, especially given the global endeavor toward higher accuracy in geodesy. This study investigates the influence of OTL near Taiwan by applying global OTL correction models to GPS relative static positioning. Hopefully, the results of this study will contribute the high precision GPS positioning in Taiwan.

\section{THEORIES OF OCEAN TIDAL LOADING AND CORRECTION METHOD}

The OTL displacement at a given site is largely related to its coordinates and its relative position among celestial bodies. The potential of tide-generating forces from the Sun and the Moon can be divided into 505 harmonics. Because of the limited data, only eleven components (M2, S2, N2, K2, K1, O1, P1, Q1, MF, MM and SSA) are considered for correction in this study. Tidal prediction methods can be categorized into harmonic or non-harmonic analyses, of which the former is more precise. Additionally, the rise and fall of sea levels are influenced by a combination of various tidal constituents, and each tidal constituent has its own period and phase. Including more tidal constituents in a sea level calculation produces a more accurate result. Table 1 lists the most influential tidal constituents at the SHJU tide gauge station in western Taiwan. The tidal components can be divided according to their periods into semi-diurnal tides, diurnal tides, fortnightly tides, monthly tides, and semi-annual tides. The different tidal constituents are computed and combined to accurately predict the future tides. OTL also influences crust movement, explaining why GPS positioning coordinates vary slightly with the tides.

In addition to determining how OTL influences local gravity, Green's function and tidal height integration methods can be used to estimate the magnitude of the effects of OTL on GPS positioning (Farrell 1972). Compared to

Table 1. Major tidal harmonic components at the SHJU tide gauge station.

\begin{tabular}{lcccc}
\hline Tide & Symbol & Period (hour) & Amplitude (mm) & Phase (degree) \\
\hline Semi-diurnal tide & & & & \\
Lunar semi-diurnal tide & M2 & 12.42 & 1632.95 & 221.87 \\
Solar semi-diurnal tide & S2 & 12.00 & 471.35 & 359.70 \\
Lunar elliptic semi-diurnal tide & N2 & 12.66 & 302.41 & 102.73 \\
Lunar-solar semi-diurnal tide & K2 & 11.97 & 129.64 & 249.19 \\
Diurnal tide & & & & \\
Luni-solar diurnal tide & K1 & 23.93 & 232.06 & 291.03 \\
Lunar diurnal tide & O1 & 25.82 & 192.98 & 86.02 \\
Solar diurnal tide & P1 & 24.07 & 73.32 & 217.37 \\
Long period tide & & & & 258.40 \\
Lunar fortnightly tide & MF & 327.86 & 7.08 & 58.04 \\
Lunar monthly tide & MM & 661.30 & 4.71 & 232.14 \\
$\quad$ Solar semi-annual tide & SSA & 4382.90 & 22.01 & \\
\hline
\end{tabular}


the method of spherical harmonics, this method is flexible with respect to the joint use of local and global tidal models and in adapting a local, detailed coastline for loading computations. At any point $p\left(\theta_{p}, \lambda_{p}\right)$ on the Earth (where is the co-latitude, and $\lambda_{p}$ is the longitude), the gravitational change $\left(L_{g}\right)$ and site displacements $\left(L_{r}, L_{\theta}\right.$, and $L_{\lambda}$ in the radial, northward and eastward directions, respectively) due to OTL are (Farrell 1972):

$$
\begin{aligned}
& L_{g}\left(\theta_{p}, \lambda_{p}\right)=\frac{g}{M} \iint_{s} \frac{\sigma(\theta, \lambda)}{4 \sin \frac{\psi}{2}} d s-\iint_{s} \sigma(\theta, \lambda) G(\psi) d s \\
& L_{r}\left(\theta_{p}, \lambda_{p}\right)=\iint_{s} \sigma(\theta, \lambda) U(\psi) d s \\
& L_{\theta}\left(\theta_{p}, \lambda_{p}\right)=\iint_{s} \sigma(\theta, \lambda) V(\psi) \cos A d s \\
& L_{\lambda}\left(\theta_{p}, \lambda_{p}\right)=\iint_{s} \sigma(\theta, \lambda) V(\psi) \sin A d s
\end{aligned}
$$

where $d s=\cos \theta d \theta d \lambda$ is the surface element, $A$ is the azimuth, $\psi$ is the spherical distance between the point $p$ and a running point at $(\theta, \lambda)$, and $\sigma(\theta, \lambda)=\rho_{0} H(\theta, \lambda)$ is the surface density of the loading mass, with $\rho_{0}$ and $H$ representing the average density of seawater $\left(1.03 \mathrm{~g} \mathrm{~cm}^{-3}\right)$ and the tidal height, respectively. The kernel functions (Green's functions) in Eqs. (1) to (4) are defined as follows (Farrell 1972):

$$
\begin{aligned}
G(\psi)= & \frac{g}{M} \sum_{n=0}^{\infty}\left[2 h_{n}^{\prime}-(n+1) k_{n}^{\prime}\right] P_{n}(\cos \psi) \\
U(\psi)= & \frac{R h_{\infty}^{\prime}}{M} \sum_{n=0}^{\infty} P_{n}(\cos \psi)+\frac{R}{M} \sum_{n=0}^{\infty}\left(h_{n}^{\prime}-h_{\infty}^{\prime}\right) P_{n}(\cos \psi) \\
V(\psi)= & \frac{R l_{\infty}^{\prime}}{M} \sum_{n=1}^{\infty} \frac{1}{n} \frac{d P_{n}(\cos \psi)}{d \psi} \\
& +\frac{R}{M} \sum_{n=1}^{\infty}\left(n l_{n}^{\prime}-l_{\infty}^{\prime}\right) \frac{1}{n} \frac{d P_{n}(\cos \psi)}{d \psi}
\end{aligned}
$$

where $k_{n}^{\prime}, l_{n}^{\prime}, h_{n}^{\prime}$ are the loading Love numbers of degree $n$, $R$ is the average Earth radius, $M$ is the average Earth mass, $g$ is the mean value of gravitational acceleration at the Earth's surface, and $P_{n}(\cos \psi)$ and $d P_{n} / d \psi$ are the Legendre function and its derivative, respectively.

First, this study analyzes data obtained during 2004 at TNML, which is an IGS (International GNSS Service) tracking station in Hsinchu. Each day is taken to be one unit of data, and each calculation is performed twice under two different sets of assumptions. We utilized the Bernese 5.0 software (Dach et al. 2007) to independently analyze the doubly-differenced phase data (the reference station is the
WULI station in central Taiwan) and obtain the coordinates of TNML. During the first calculation, OTL is estimated with the NAO.99b model, whereas OTL is left uncorrected in the second calculation. In other words, we compute the coordinates of TNML with and without an OTL correction. Moreover, the ambiguities fixed to the same values by GPS data processing with and without an OTL correction. Figure 1 summarizes the differences between the vertical GPS coordinates obtained from these two calculations. According to this figure, the vertical coordinates of TNML change cyclically, with a period of approximately 15 days and an amplitude as high as $2 \mathrm{~cm}$. This variation can be attributed to the influence of un-modeled semi-diurnal tides (Penna et al. 2007). Thus, for users who require precise vertical GPS positioning, the errors caused by OTL must be taken into account and corrected, especially on an island such as Taiwan.

The more influential semi-diurnal tidal constituents in Taiwan are the M2 lunar semi-diurnal tides, the N2 lunar elliptic tides and the $\mathrm{S} 2$ solar semi-diurnal tides. The diurnal tidal constituents include the K1 lunar-solar diurnal tides and the $\mathrm{O} 1$ lunar diurnal tides. The magnitudes of the M2 tidal constituents significantly increase between Keelung and Taipei, peak in Taichung, begin to decline further south and decrease sharply in Tainan. The N2 and S2 semi-diurnal tides behave similarly. However, the $\mathrm{K} 1$ and $\mathrm{O} 1$ diurnal tides exhibit no such trend. The range of tidal amplitudes is smallest in southern Taiwan and greatest in central Taiwan, with northern Taiwan falling in between the two. The average tide range is $54.3 \mathrm{~cm}$ in Keelung, $220.0 \mathrm{~cm}$ in Taipei, $253.9 \mathrm{~cm}$ in Taoyuan, $349.5 \mathrm{~cm}$ in Hsinchu, $367.6 \mathrm{~cm}$ in Taichung, $214.6 \mathrm{~cm}$ in Yunlin, $145.9 \mathrm{~cm}$ in Chiayi, $49.2 \mathrm{~cm}$ in Kaohsiung and $96.2 \mathrm{~cm}$ in Taitung.

Because tidal variations can be regarded as the sum of the set of known tidal frequencies and amplitudes, each

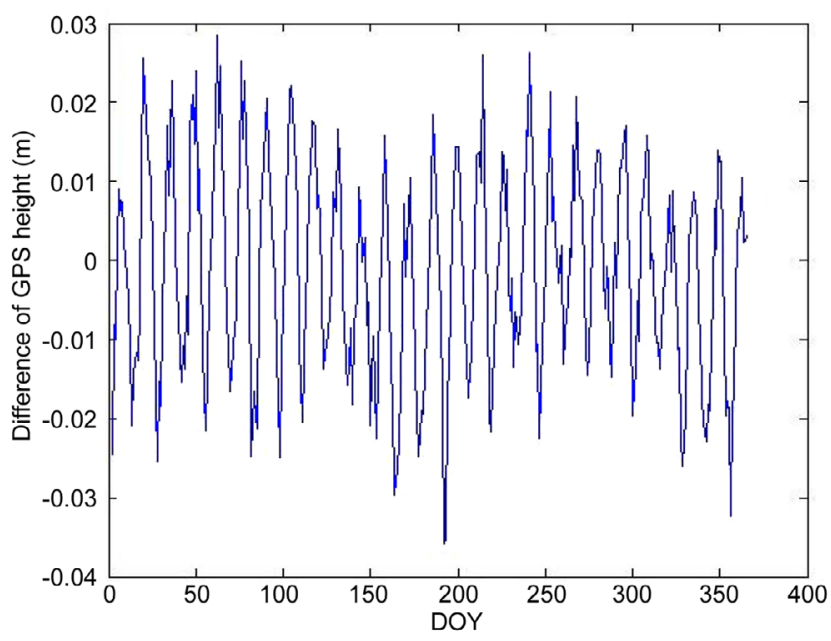

Fig. 1. Differences between GPS heights with and without OTL correction with the NAO.99b model at the TNML station in 2004. 
tidal constituent can be expressed as a harmonic function, as shown in following formula:

$y(t)=\sum_{i=1}^{N} A_{i} \cos \left[v_{i}(t)-\phi_{i}\right]$

where $A_{i}$ denotes the amplitude, $\phi_{i}$ represents the phase, and $v_{i}(t)$ is an astronomical argument. A least-squares analysis is then performed to divide the data into several components (i) to obtain the harmonic functions representing the major tidal constituents of a tidal wave. The essential assumption of harmonic analysis is that the time series of the data is periodic and is composed of harmonic functions of a limited number of known frequencies. In this study, four models are chosen to conduct surveys: Schwiderski, NAO.99b, CSR4.0, and TPXO.6.2 (see Table 2).

\section{GPS DATA PROCESSING}

Since GPS precise position estimates are obtained from relative positioning, a GPS station must be selected as reference which is not or only nominally influenced by the OTL. Since the effects of OTL are smaller for stations further from the sea, the WULI station in central Taiwan is selected as the reference station for this study. We assume that the effects of OTL at this station are very small after proper correction and can be neglected.

The test data are measured during 8 - 14 August 2006 and 1 - 30 January 2007. The Bernese 5.0 software, which was created by the University of Bern, Switzerland, was adopted for data processing (Dach et al. 2007). We analyzed GPS data using different strategies for troposphere correction as a baseline solution. Phase ambiguities were solved and fixed to integer values. Pole tide and solid earth tide corrections were applied. Precise IGS satellite orbits were used and kept fixed. A cutoff angle of 10 degrees was selected to limit tropospheric delay (Yeh et al. 2010). We applied an a priori Saastamoinen model (Saastamoinen 1973) to correct the tropospheric influence in the point positioning. Moreover, we estimated the ZTD parameters of each day using the Saastamoinen a priori delays and the $1 / \cos (Z)$ mapping function ( $Z$ is the zenith angle). Furthermore, we used ionosphere-free linear combination to simultaneously estimate the site coordinates and ZTD. Hence, the site coordinates were corrected for the troposphere by applying the ZTD parameters (Yeh et al. 2009; Chen et al. 2011).

Moreover, the amplitudes and phases of each tide obtained from global ocean tide correction models were used to analyze the positioning accuracy. Site coordinates were computed using 1-hour, 2-hour and 3-hour data intervals. We obtained a high sampling resolution with 1-hour intervals, but having fewer data produced the poorer accuracy. Therefore, the site coordinates were computed on a 3-hour basis to balance high sampling resolution and high positioning accuracy. The resulting three-dimensional coordinates were used to analyze the coordinate variations caused by short-period OTL (such as semi-diurnal tides and diurnal tides).

\section{OTL EFFECTS ON GPS POSITIONING PRECI- SION AROUND TAIWAN}

Experiments were performed in various places within Taiwan and its offshore islands. Figure 2 presents the distribution of observation stations. Data with a sampling rate of 30 seconds were obtained from 1 - 30 January 2007. The data were divided into three-hour subsets and processed, resulting in eight sets of coordinates for each site for each day, or 240 coordinate estimates for the entire month. At this point of the data processing, no OTL corrections were applied so that OTL's influence on the precision of GPS positioning in Taiwan can be evaluated. Harmonic analysis with a tidal period of 12.42 hours was performed on the 240 vertical coordinates (the M2 tidal constituent lasts 12.42 hours) to determine the amplitude and phase of each M2 tidal constituent at each station. Figure 3 shows the vertical amplitudes at each station and indicates that the amplitudes on the northwestern coast exceed those on the southeastern coast of Taiwan. The vertical amplitudes measured throughout all of Taiwan ranged from 0.28 to $2.7 \mathrm{~cm}$.

Table 2. The tidal models used in this study.

\begin{tabular}{|c|c|c|c|}
\hline Model & Area & Resolution & Type of solution \\
\hline $\begin{array}{l}\text { Schwiderski } \\
\text { (Schwiderski 1980) }\end{array}$ & Global & 1 by 1 degree grid & $\begin{array}{l}\text { Hydrodynamic interpolation model, global fit of tide } \\
\text { gauges }\end{array}$ \\
\hline $\begin{array}{l}\text { NAO.99b } \\
\text { (Matsumoto et al. 2000) }\end{array}$ & Global & 0.5 by 0.5 degree grid & $\begin{array}{l}\text { Based on the same hydrodynamics model as the } \\
\text { Schwiderski model, but has TOPEX/Poseidon data } \\
\text { incorporated into it }\end{array}$ \\
\hline $\begin{array}{l}\text { CSR4.0 } \\
\text { (Eanes et al. 1996) }\end{array}$ & Global & 0.5 by 0.5 degree grid & $\begin{array}{l}\text { Long wavelength adjustments of FES } 94.1 \text { using } \\
\text { TOPEX/Poseidon data. Spurious grid-cells over land } \\
\text { have been removed }\end{array}$ \\
\hline $\begin{array}{l}\text { TPXO.6.2 } \\
\text { (Egbert et al. 2002) }\end{array}$ & Global & 0.25 by 0.25 degree grid & $\begin{array}{l}\text { Based on TPXO. } 5 \text {, but improved the resolution to } \\
0.25 \text { by } 0.25 \text { degree grid }\end{array}$ \\
\hline
\end{tabular}


The topography and depth distributions of the ocean floors around Taiwan (Fig. 4) explain this observation. In eastern Taiwan, the vertical amplitudes at all GPS stations are smaller than $1 \mathrm{~cm}$ because the ocean is more than $2000 \mathrm{~m}$ deep and with shallower and narrower bights and estuaries. We can compare these results with the tide gauge data described in section 2 . The average tide ranges in eastern Taiwan are less than $100 \mathrm{~cm}$, which agrees with the distribution of GPS height variations. On the other hand, the amplitudes of northwestern GPS stations are larger than $1 \mathrm{~cm}$ because the ocean is only around $200 \mathrm{~m}$ deep in this area and the average tide ranges in this area are very large (up to $370 \mathrm{~cm}$ ).

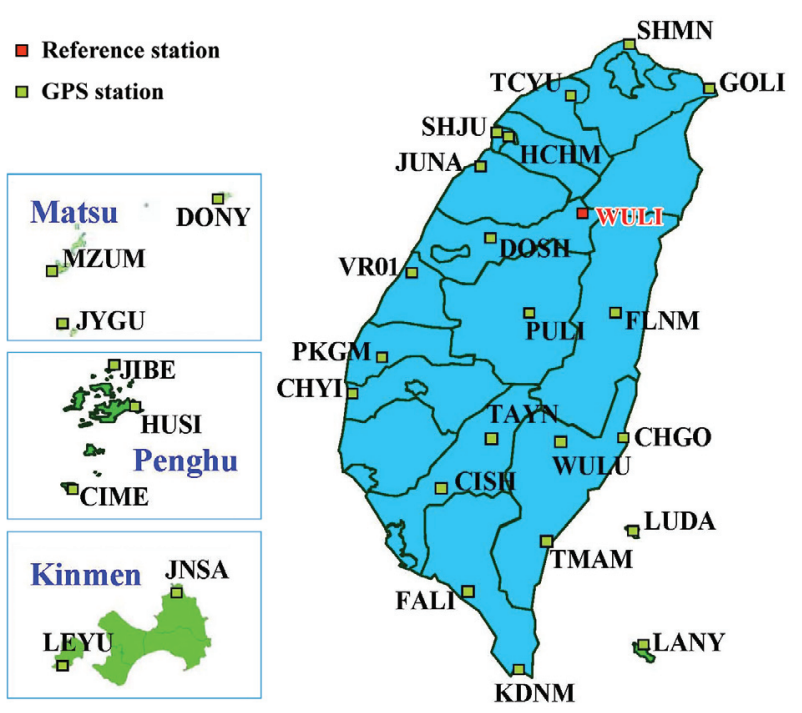

Fig. 2. Positions of the referential GPS station (red square) and the tested GPS stations (green squares) in Taiwan and on the offshore islands of Taiwan.

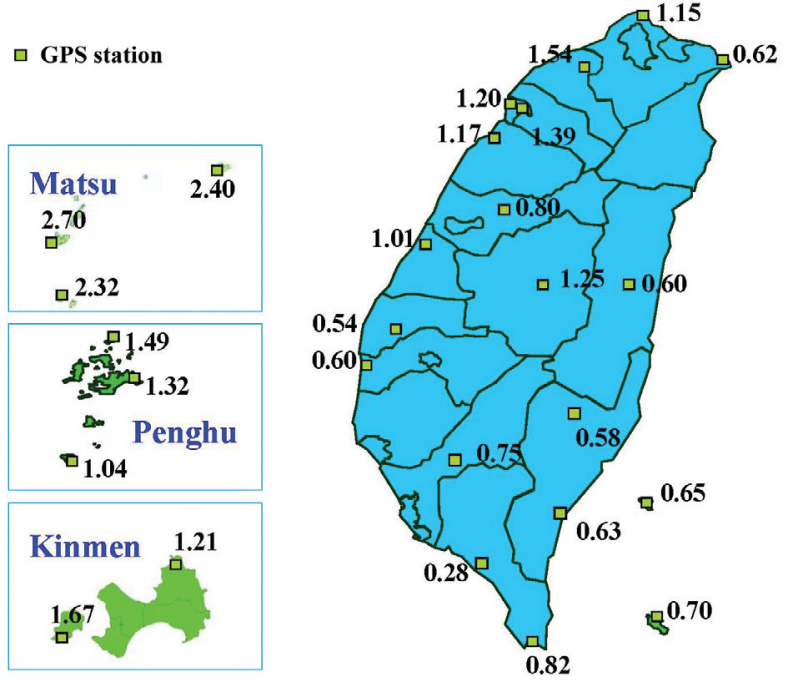

Fig. 3. Distribution of GPS position variation amplitudes (in $\mathrm{cm}$ ) from the M2 tide at each GPS tracking station.
The amplitudes of GPS stations on the offshore islands of Taiwan are around $1.2-2.7 \mathrm{~cm}$, larger than those of stations within Taiwan on average. The largest amplitudes are observed at Matsu Island, which is very close to mainland China and exhibits GPS height variations of $2.3-2.7 \mathrm{~cm}$.

Figure 5 shows the phase distributions of the vertical coordinate variations caused by lunar semi-diurnal tides at various stations. As the M2 tidal constituent lasts 12.42 hours, each hour is roughly equivalent to a 30 -degree phase delay. The tides proceed from the Pacific Ocean to the Asian continent, causing an initial rise at the Green Island station in the east offshore area of Taiwan. The tides gradually rise toward the north and the east and also rise significantly between the JUNA and CHYI stations on the western shore. Thus, lunar semi-diurnal tides tend to gather in central Taiwan from the northern and southern tips of the Taiwan Strait, with the greatest tidal heights found at the Penghu and Kinmen stations. In other words, the tides rise latest at the two mentioned stations, with an average phase delay of approximately 120 degrees or about 4 hours.

\section{APPLICABILITY OF GLOBAL OTL MODELS IN TAIWAN}

This section describes how OTL affects GPS positioning at the GPS tracking stations on Taiwan and its offshore

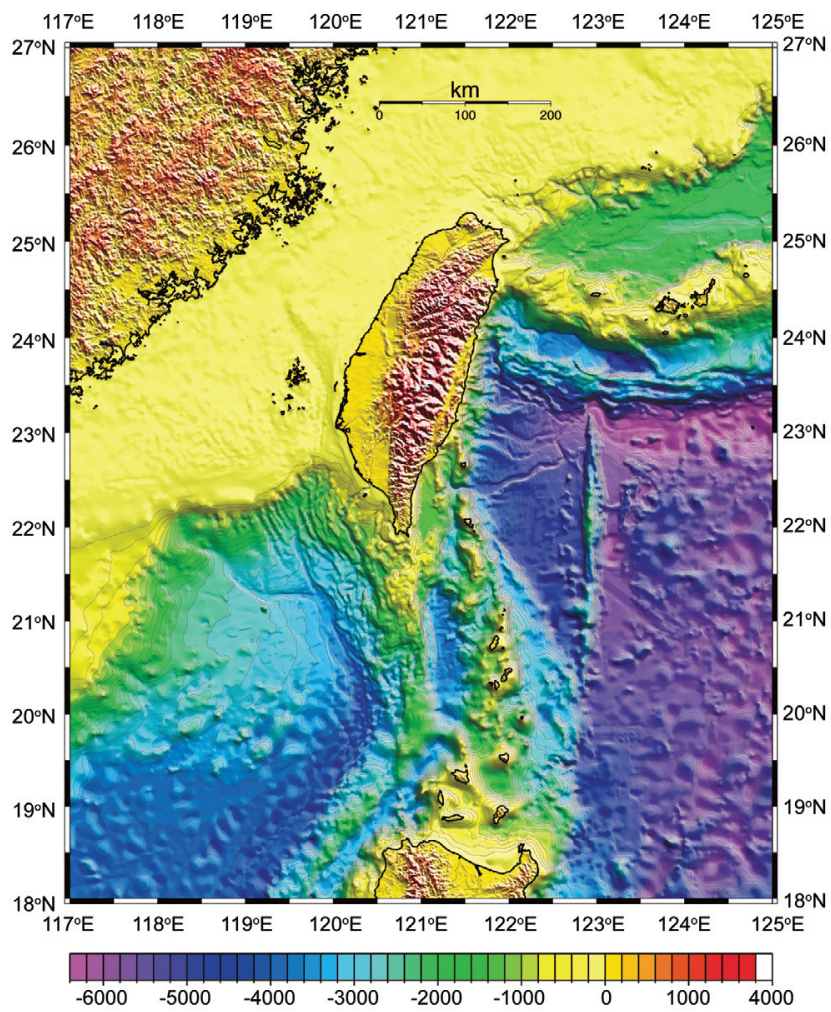

Fig. 4. The depth of surrounding sea areas and the topography of the ocean floors surrounding Taiwan. 


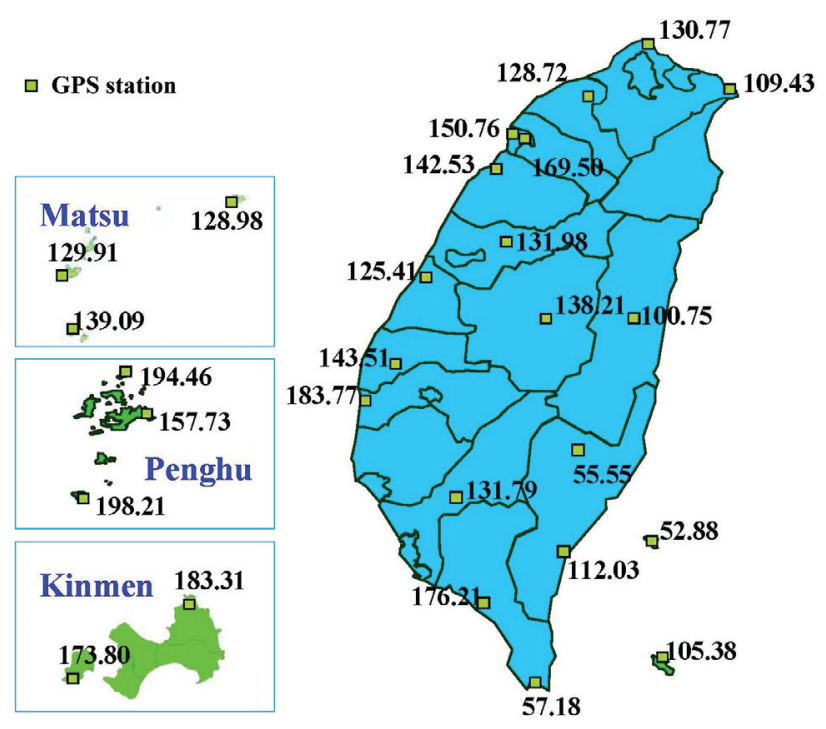

Fig. 5. Distribution of the GPS vertical phases (in degrees) of the M2 tide at each GPS tracking station.

islands. After basic processing without OTL corrections, corrections derived from ocean tide models were applied to evaluate their effect on the resulting site coordinates. Four OTL models were adopted here: Schwiderski, NAO.99b, CSR4.0, and TPXO.6.2. Table 2 summarizes the contents of these models. The observational data were divided into summer and winter groups: 8 - 14 August 2006 (summer) and 24 - 30 January 2007 (winter).
Bernese 5.0 software is again utilized for data processing, with calculations performed on 3-hour-long sets of data. Fifty-six position coordinates were obtained over each seven-day period. Numerous stations were studied, but only the vertical coordinates of the JUNA station on the western coast of Taiwan will be discussed in detail. Figure 6 summarizes the vertical coordinate variations as estimated through four OTL corrections. Figure 7 shows the results of calculations on the winter dataset, in which four OTL models are used to modify the baseline measurements at JUNA. The bold line denotes the results without corrections, and the other lines, in various types, represent the vertical coordinate variations obtained after corrections with the Schwiderski, NAO.99b, CSR4.0, and TPXO.6.2 models. According to this figure, the vertical coordinate variations of JUNA fluctuate between $45.23-45.62 \mathrm{~cm}$ in summer and 45.38 $-45.46 \mathrm{~cm}$ in winter.

The average vertical errors of the measurements above are obtained using various OTL correction models (Table 3 ). The average uncorrected error during summer is $2.72 \mathrm{~cm}$, and after performing corrections based on the Schwiderski, NAO.99b, CSR4.0 and TPXO.6.2 models, the errors changed to $2.30,2.30,2.30$ and $2.31 \mathrm{~cm}$, respectively, demonstrating improvements of $0.41-0.42 \mathrm{~cm}$ in the vertical positioning precision. The average uncorrected error during winter is $1.32 \mathrm{~cm}$, and corrections based on the four models reduced the errors to $0.86,0.85,0.89$, and $0.84 \mathrm{~cm}$, respectively, improving the precision by $0.43-0.48 \mathrm{~cm}$. The results of all four models differ only slightly. Although the

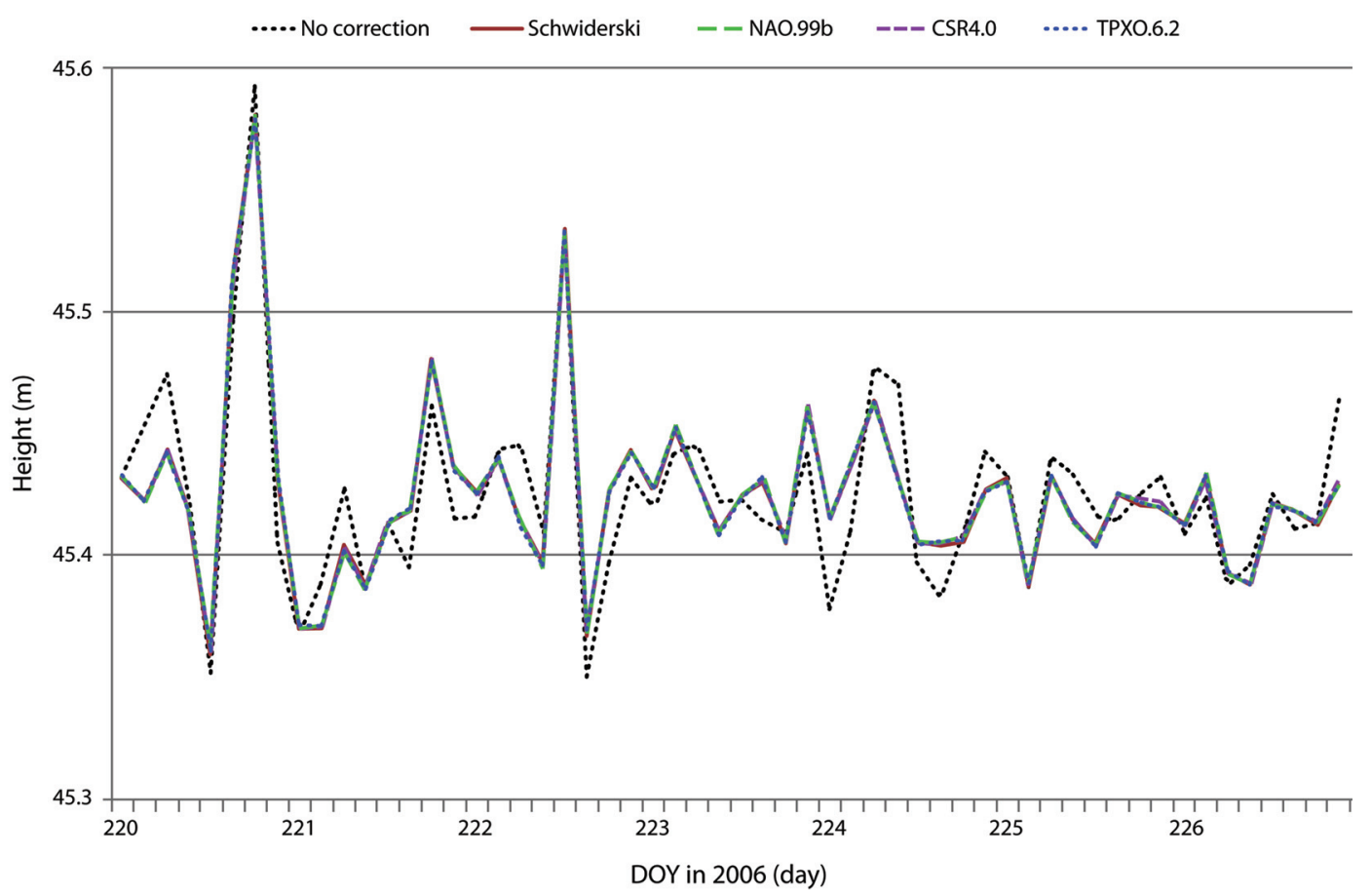

Fig. 6. GPS height variations after different OTL corrections in summer at the JUNA station. 


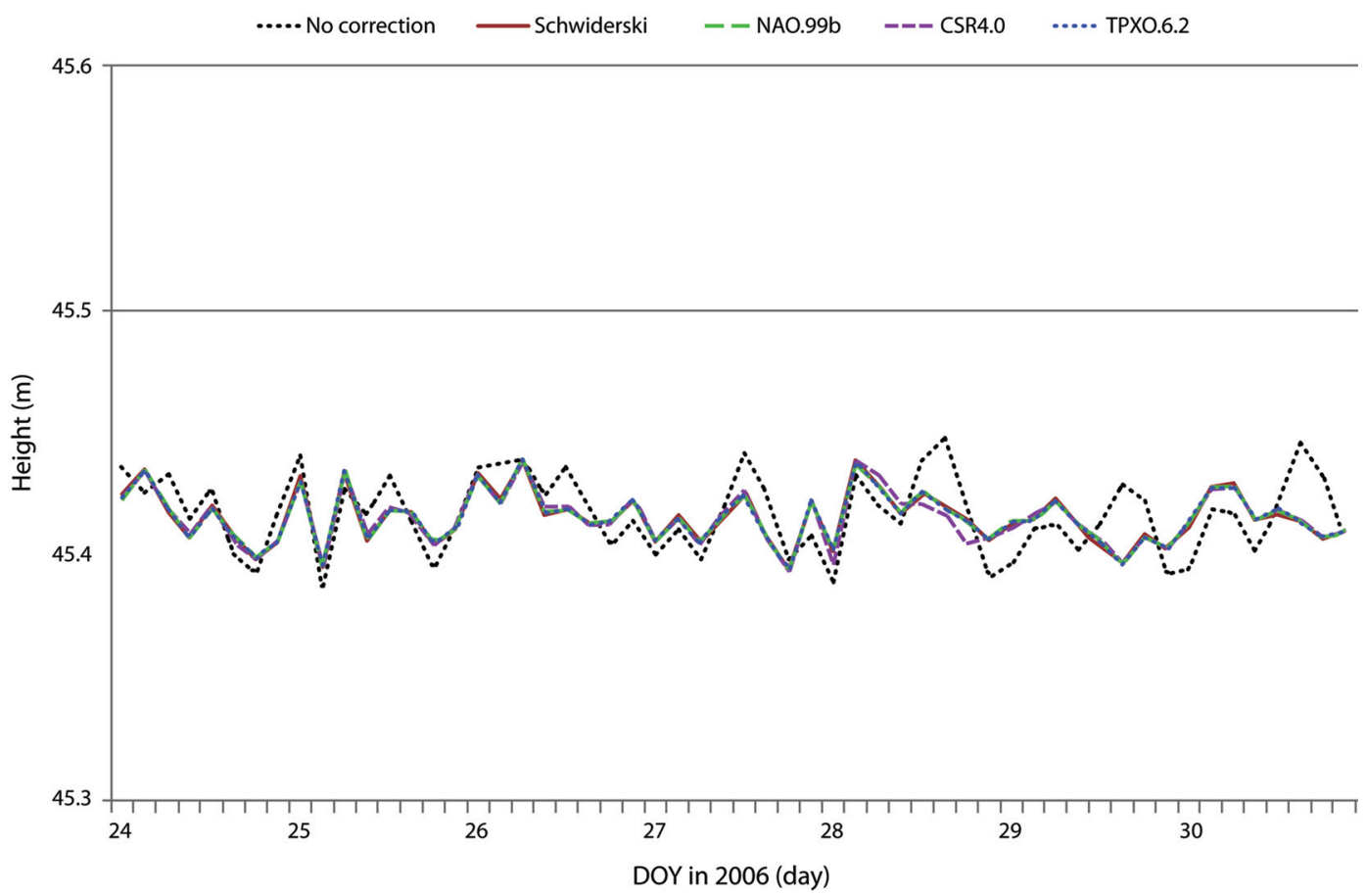

Fig. 7. GPS height variations after different OTL corrections in winter at the JUNA station.

Table 3. GPS height errors from different OTL corrections in summer and in winter at the JUNA station.

\begin{tabular}{|c|c|c|c|c|c|}
\hline \multirow{2}{*}{ Station } & \multirow{2}{*}{ Model } & \multicolumn{2}{|c|}{ In summer } & \multicolumn{2}{|c|}{ In winter } \\
\hline & & Average height (m) & Average error $(\mathbf{c m})$ & Average height (m) & Average error $(\mathrm{cm})$ \\
\hline \multirow{5}{*}{ JUNA } & No correction & 45.4251 & 2.72 & 45.4172 & 1.32 \\
\hline & Schwiderski & 45.4248 & 2.30 & 45.4157 & 0.86 \\
\hline & NAO.99b & 45.4249 & 2.30 & 45.4157 & 0.85 \\
\hline & CSR4.0 & 45.4249 & 2.30 & 45.4155 & 0.89 \\
\hline & TPX0.6.2 & 45.4249 & 2.31 & 45.4157 & 0.84 \\
\hline
\end{tabular}

models can improve the vertical positioning accuracy by 15 to $36 \%$ on average, NAO.99b appears to produce slightly better results than the other three models in these cases.

Exactly how much the precision of OTL corrections differ during summer and winter was further analyzed by using only the NAO.99b model for correction. The 56 uncorrected vertical coordinates estimated at each site were subjected to a harmonic analysis to extract any signal with a period equivalent to that of the M2 tidal harmonic. Although numerous stations were studied, only the vertical coordinates of the VR01 station on the western coast of Taiwan are presented here. Figure 8 illustrates the vertical coordinate variations during summer derived from least-squares adjustment with harmonic analysis. Figure 9 summarizes similar results from the winter. The initial vertical GPS coordinates at VR01 fall between $39.34-39.54 \mathrm{~cm}$ during summer and between $39.38-39.46 \mathrm{~cm}$ during winter. The variation is larger in summer than winter. However, the results of harmonic analysis are generally equivalent during summer and winter. The amplitude is around $2 \mathrm{~cm}$ after adjustment, indicating that the OTL effects caused by M2 tides remain constant with the seasons. Exactly why the initial vertical coordinate variations are greater during summer is further explored by exploring the typhoon database of the Central Weather Bureau of Taiwan. During the summer observation period, from 7 - 10 August, the Baofa and Sunmei typhoons struck Taiwan, producing a great deal of rainfall islandwide. During the typhoon, drastic humidity changes may have resulted in imperfect tropospheric delay corrections when the baseline was processed, explaining the increased errors in the GPS vertical coordinates. Moreover, water on the antenna, storm surge, atmospheric loading, and water loading on land, all associated with the typhoons, may influence the GPS precision. Figure 8 shows that the amplitudes became 


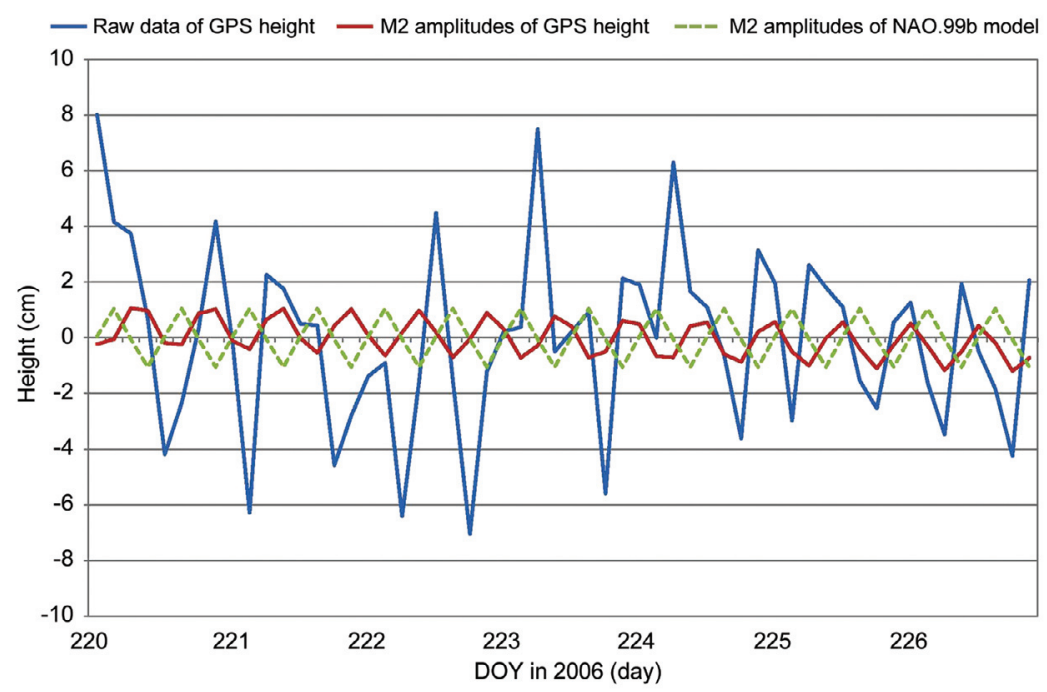

Fig. 8. Comparison of raw GPS height data (blue lines), M2 amplitudes obtained from a least-squares adjustment of GPS height with harmonic analysis (red lines) and M2 amplitudes from the NAO.99b model (dotted lines) in summer at the VR01 station.

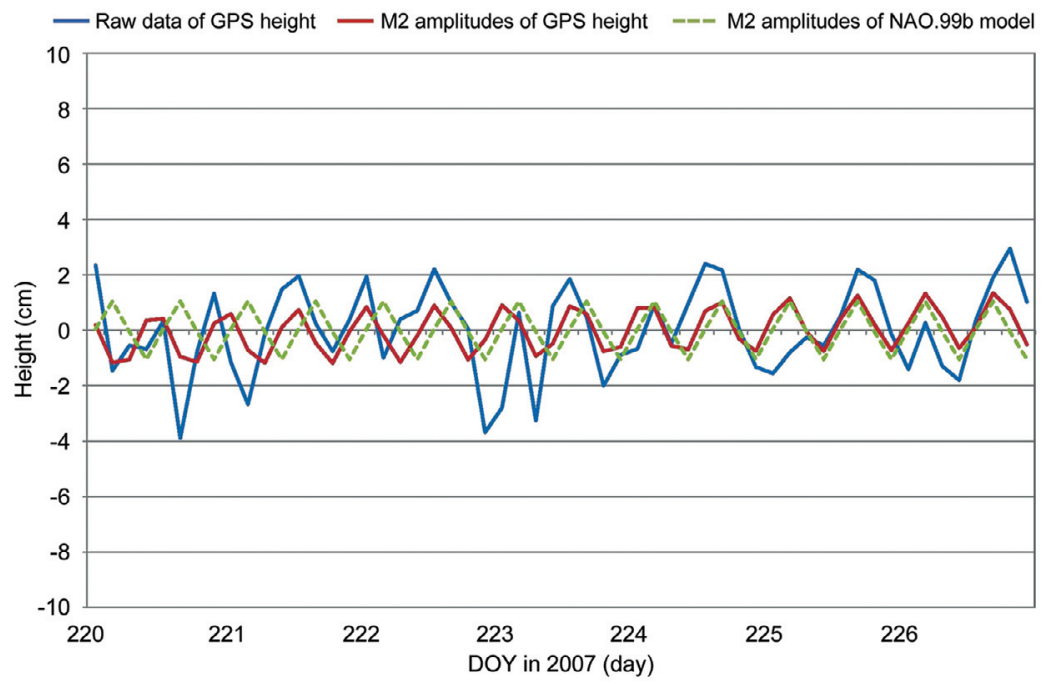

Fig. 9. Comparison of raw GPS height data (blue lines), M2 amplitudes obtained from a least-squares adjustment of GPS height with harmonic analysis (red lines) and M2 amplitudes from the NAO.99b model (dotted lines) in winter at the VR01 station.

smoother after 12 August (DOY 224), indicating that the typhoons had left the sea areas surrounding Taiwan.

\section{CONCLUSIONS}

In this study, variations of the GPS vertical coordinate are used to discuss the influence of OTL on GPS positioning. We discovered that the amplitudes at the northwestern coast of Taiwan are greater than those at the southeastern coast. The vertical amplitudes measured throughout all of Taiwan ranged from 0.28 to $2.7 \mathrm{~cm}$. This distribution of amplitudes is attributed to the depth of the surrounding sea areas and the topography of ocean floors around Taiwan. Corrections preformed using four different OTL models improve the precision of the vertical GPS position measurements by $15-36 \%$ on average. Of the four models tested, NAO.99b produces slightly better results than the other three. In eastern Taiwan, the amplitudes of all GPS stations are smaller than $1 \mathrm{~cm}$ because the ocean is over $2000 \mathrm{~m}$ deep. The amplitudes of northwestern GPS stations are larger than $1 \mathrm{~cm}$ because the ocean is only around $200 \mathrm{~m}$ deep in this region. The amplitudes of GPS stations in the offshore islands of Taiwan are around $1.2-2.7 \mathrm{~cm}$, which are larger than those within Taiwan on average. Analyzing OTL corrections in different seasons revealed that the initial GPS vertical positions measured at the VR01 station in western Taiwan range from $39.34-39.54 \mathrm{~cm}$ in summer and $39.38-39.46 \mathrm{~cm}$ in winter. It seems that corrections are not as effective in the 
summer as in the winter. However, the results of harmonic analysis during the summer and winter are comparable. The amplitude is reduced to about $2 \mathrm{~cm}$ after adjustment, suggesting that vertical variations resulting from M2 tides do not change with the seasons.

In the dataset utilized above, some of the OTL effects can be corrected. Further, a $4-5 \mathrm{~mm}$ positioning accuracy can be improved. This improved position precision may be useful in some fields such as regional engineering surveys and/or cadastral surveys. However, the residual errors from OTL effects in Taiwan are still too large to detect slight variations such as fault movement and geodynamics. In the future, a local OTL model will be necessary for highly accurate positioning. Moreover, through GPS observation on the tide gauge station to modify the coastal OTL model. Using such modified OTL model may be able to reduce the errors to a more precise level. If we can clearly reduce the effects of OTL and further enhance the accuracy of GPS measurements, this technology can be used in a wide range of applications in Taiwan. Moreover, the precision of baseline processing is unsatisfactory, indicating that GPS errors caused by other factors are still large. We suggest that, in the future, tropospheric delay correction methods should be improved, and GPS data obtained from longer consecutive periods should be studied to obtain more tidal periods in the dataset and support the findings of this research.

Acknowledgements The authors would like to thank the National Science Council of the Republic of China, Taiwan, for financially supporting this research under Contract No. NSC94-2218-E-231-001.

\section{REFERENCES}

Baker, T. F., D. J. Curtis, and A. H. Dodson, 1995: OTL and GPS, GPS World, 54-59.

Bos, M. S. and H. G. Scherneck, 2007: OTL provider. Onsala Space Observatory, available at http://www.oso. chalmers.se/ loading/.

Chen, C. H., T. K. Yeh, J. Y. Liu, C. H. Wang, S. Wen, H. Y. Yen, and S. H. Chang, 2011: Surface deformation and seismic rebound: Implications and applications. Surv. Geophys., 32, 291-313, doi: 10.1007/s10712-01 1-9117-3. [Link]

Dach, R., U. Hugentobler, P. Fridez, and M. Meindl, 2007: Bernese GPS Software Version 5.0 User Manual, Astronomical Institute University of Bern, Bern, Switzerland.

Dragert, H., T. S. James, and A. Lambert, 2000: Ocean loading corrections for continuous GPS: A case study at the Canadian coastal site Holberg. Geophys. Res. Lett., 27, 2045-2048.

Eanes, R. J. and S. Bettadpur, 1996: The CSR3.0 global ocean tide model: Diurnal and semi-diurnal ocean tides from TOPEX/POSEIDON altimetry, CRS-TM-96-05, University of Texas, Centre for Space Research, Austin, Texas.

Egbert, G. D. and S. Y. Erofeeva, 2002: Efficient inverse modeling of barotropic ocean tides. J. Atmos. Ocean. Technol., 19, 183-204, doi: 10.1175/1520-0426(2002) 019<0183:EIMOBO>2.0.CO;2. [Link]

Farrell, W. E., 1972: Deformation of the earth by surface loads. Rev. Geophys., 10, 761-797, doi: 10.1029/RG01 0i003p00761. [Link]

Jentzsch, G., P. Knudsen, and M. Ramatschi, 2000: Ocean tidal loading affecting precise geodetic observations on Greenland: Error account of surface deformations by tidal gravity measurements. Phys. Chem. Earth (A), 25, 401-407, doi: 10.1016/S1464-1895(00)00063-6. [Link]

Khan, S. A. and H. G. Scherneck, 2003: The $M_{2}$ ocean tide loading wave in Alaska: Vertical and horizontal displacements, modelled and observed. J. Geodesy, 77, 117-127, doi: 10.1007/s00190-003-0312-y. [Link]

Matsumoto, K., T. Takanezawa, and M. Ooe, 2000: Ocean tide models developed by assimilating TOPEX/POSEIDON altimeter data into hydrodynamical model: A global model and a regional model around Japan. $J$. Oceanogr., 56, 567-581, doi: 10.1023/A:1011157212 596. [Link]

Penna, N. T. and T. F. Baker, 2002: Ocean tide loading considerations for GPS processing around Australia. Geomatics Research Australasia, 77, 1-26.

Penna, N. T., M. A. King, and M. P. Stewart, 2007: GPS height time series: Short-period origins of spurious long-period signals. J. Geophys. Res., 112, B02402, doi: 10.1029/2005JB004047. [Link]

PO.DAAC, 2007: TOPEX/POSEIDON tides models. Physical Oceanography Distributed Active Archive Center.

Saastamoinen, J., 1973: Contributions to the theory of atmospheric refraction. Part II. Refraction corrections in satellite geodesy. Bull. Geodesique, 107, 13-34, doi: 10.1007/BF02522083. [Link]

Schwiderski, E. W., 1980: On charting global ocean tides. Rev. Geophys., 18, 243-268, doi: 10.1029/RG018i00 1p00243. [Link]

van Dam, T., H. P. Plag, O. Francis, and P. Gegout, 2003: GGFC special bureau loading: Current status and plans. In: Richter, B., W. Schwegmann, and W. R. Dick (Eds.), Proceedings of the IERS Workshop on Combination Research and Global Geophysical Fluids, Bavarian Academy of Sciences, Munich, Germany, 18-21 November 2002, International Earth Rotation and Reference Systems Service (IERS), IERS Technical Note, 30, 180-198.

Xu, G., 2007: GPS: Theory, Algorithms and Applications. $2^{\text {nd }}$ edition, Springer Heidelberg, ISBN 978-3-54072714-9. 
Yeh, T. K., C. Hwang, and G. Xu, 2008: GPS height and gravity variations due to ocean tidal loading around Taiwan. Surv. Geophys., 29, 37-50, doi: 10.1007/s107 12-008-9041-3. [Link]

Yeh, T. K., C. Hwang, G. Xu, C. S. Wang, and C. C. Lee, 2009: Determination of global positioning system (GPS) receiver clock errors: Impact on positioning accuracy. Meas. Sci. Technol., 20, 075105, doi: 10.1088/0957-0233/20/7/075105. [Link]
Yeh, T. K., Y. J. Chen, Y. D. Chung, C. W. Feng, and G. $\mathrm{Xu}, 2010$ : Clarifying the relationship between quality of Global Positioning System data and precision of positioning. J. Surv. Eng., 136, 41-45, doi: 10.1061/(AS CE)SU.1943-5428.0000001. [Link]

Zahran, K. H., G. Jentzsch, and G. Seeber, 2005: Worldwide synthetic tide parameters for gravity and vertical and horizontal displacements. J. Geodesy, 79, 293-299, doi: 10.1007/s00190-005-0460-3. [Link] 